\title{
Importance of immunogenicity testing for cost-effective management of psoriasis patients treated with adalimumab
}

\author{
Fernando Mota ${ }^{1 凶}$, Esmeralda Neves², José Carlos Oliveira ${ }^{3}$, Manuela Selores $^{1,4,5}$, Tiago Torres ${ }^{1,4,5}$
}

\begin{abstract}
Introduction: Up to $30 \%$ of patients treated with anti-tumor necrosis factor drugs do not respond adequately, and up to $50 \%$ lose response over time. Immunogenicity is now known to be one of the main causes of this loss of response.

Methods: Serum levels of adalimumab and anti-drug antibodies (ADAs) were measured in 19 patients with psoriasis.

Results: Eighty-nine percent of the patients were responders (Psoriasis Area Severity Index (PASI) > 75) and $11 \%$ were partial responders (PASI 50-75). The serum levels of adalimumab were lower than the cutoff in both of the partial responders and the ADAs were high, whereas the other 17 patients had adalimumab levels above the cutoff and low ADA levels. Both partial responders were obese and none of them were taking methotrexate. Both patients switched to ustekinumab, and a PASI 90 response was observed after 16 weeks.

Conclusion: Immunogenicity is a risk of biological drugs. In this work, the detection of low levels of adalimumab and high levels of ADAs using a sandwich ELISA correlated with loss of clinical response. Testing immunogenicity and the drug pharmacokinetics of biological drugs in psoriasis patients will probably be part of the daily management of these patients in the future.
\end{abstract}

Keywords: psoriasis, adalimumab, immunology

Received: 3 February 2017| Returned for modification: 16 March 2017 | Accepted: 27 March 2017

\section{Introduction}

The introduction of anti-tumor necrosis factor (TNF) drugs in our daily practice has revolutionized the treatment of several inflammatory diseases, including psoriasis $(1,2)$. However, up to $30 \%$ of patients treated with these drugs do not respond adequately (primary failure) and up to 50\% lose response over time (secondary failure) $(3,4)$. Immunogenicity through the induction of anti-drug-antibodies (ADA) is now known to be one of the main causes for this loss of response and may also be responsible for safety issues (5). In this setting, the evaluation of immunogenicity and drug pharmacokinetics has gained importance in recent years and might represent the key to using anti-TNF agents in a more economical, cost-effective way without losing efficacy. The enzyme-linked immunosorbent assay (ELISA) technique has been used to measure both drug and anti-drug antibody levels. ELISA is a biochemical assay that uses antibodies and an enzyme-mediated color change to detect the presence of either antigens (proteins, peptides, hormones, etc.) or antibodies in a given sample (6). A sub-type of ELISA-"sandwich" ELISA-is a more sensitive method, in which a "capture" antibody and a "detection" antibody are utilized, both binding to the peptide one wants to quantitate, but at distinct and non-overlapping epitopes, making it a more powerful tool for monitoring immunogenicity (7).

\section{Methods}

Nineteen patients with psoriasis (with or without psoriatic arthritis) treated with adalimumab were included. The adalimumab dosage was the approved dosage for psoriasis (an initial dose of $80 \mathrm{mg}$, followed by $40 \mathrm{mg}$ every other week starting 1 week after the initial dose), except for one patient, who had 2 months of double dosage (40 mg/week) after clinical worsening. The serum levels of adalimumab and ADAs were measured using the Lisa Tracker drug and anti-drug enzyme-linked immunosorbent assay (ELISA) kits for Adalimumab (Theradiag®). The samples were collected immediately before the drug administration.

\section{Results}

The characteristics of the 19 patients are shown in Table 1 . At the time of the evaluation, $89 \%(n=17)$ of the patients were responders (Psoriasis Area Severity Index (PASI) $>75)$ and $11 \%(n=2)$ were partial responders (PASI 50-75).

Table 1 | Patient characteristics. PASI = psoriasis area severity index, MTX = methotrexate.

\begin{tabular}{lc}
\hline Patient characteristics & $n=19$ \\
\hline Sex: Female & $10(52.6 \%)$ \\
Age, years (mean \pm SD) & $56 \pm 10$ \\
Psoriatic arthritis & $12(63.2 \%)$ \\
Responders (PASI > 75) & $17(89 \%)$ \\
Partial responders (PASI 50-75) & $2(11 \%)$ \\
Duration of treatment w/ adalimumab, months (mean \pm SD) & $58 \pm 23$ \\
$\quad$ Maximum & 83 \\
Minimum & 13 \\
Co-treatment w/ MTX & $5(26.3 \%)$ \\
\hline
\end{tabular}

The serum levels of adalimumab were lower than the cutoff established for the exam in both of the partial responders (PR) and the ADA levels were high (Table 2), whereas the other 17 patients had adalimumab levels above the cutoff (mean $6.6 \mu \mathrm{g} / \mathrm{ml}$ ) and low ADA levels $(<10 \mu \mathrm{g} / \mathrm{ml})$. Figure 1 shows the distribution and relation between adalimumab and ADA levels. Both PRs were obese (body mass index > 35) and none of them were taking methotrexate (MTX). One of the patients had been co-treated with acitretin after an initial worsening but had no satisfactory response after 4 
months, whereas in the other case the dose of adalimumab was doubled (40 mg weekly) for 2 months with a slight improvement but worsened after returning to the standard regimen. Both patients were switched to ustekinumab, and a PASIgo response was observed after 16 weeks.

Table 2 | Partial responder (PR) characteristics. $M T X=$ methotrexate, ADA = anti-drug antibody.

\begin{tabular}{lcc}
\hline Partial responder characteristics & PR 1 & PR 2 \\
\hline Age, years & 67 & 46 \\
Sex & Male & Female \\
Body mass index & 35 & 39 \\
Psoriatic arthritis & No & Yes \\
Co-treatment w/ MTX & No & No \\
Duration of treatment w/ adalimumab, months & & \\
$\quad$ Total & 13 & 24 \\
$\quad$ w/ partial response* & 6 & 6 \\
Duration of dose optimization, months** & 0 & 2 \\
Adalimumab levels $(\mu \mathrm{g} / \mathrm{ml})$ & 0.1 & 0.7 \\
$\quad$ Cutoff $>4.9 \mu \mathrm{g} / \mathrm{ml}$ & & \\
ADA levels $(\mu \mathrm{g} / \mathrm{ml})$ & 27.6 & 9.6 \\
$\quad$ Cutoff $>10 \mu \mathrm{g} / \mathrm{ml}$ & & \\
\hline
\end{tabular}

*From worsening until biologic switch

**Duplication of standard dose regimen

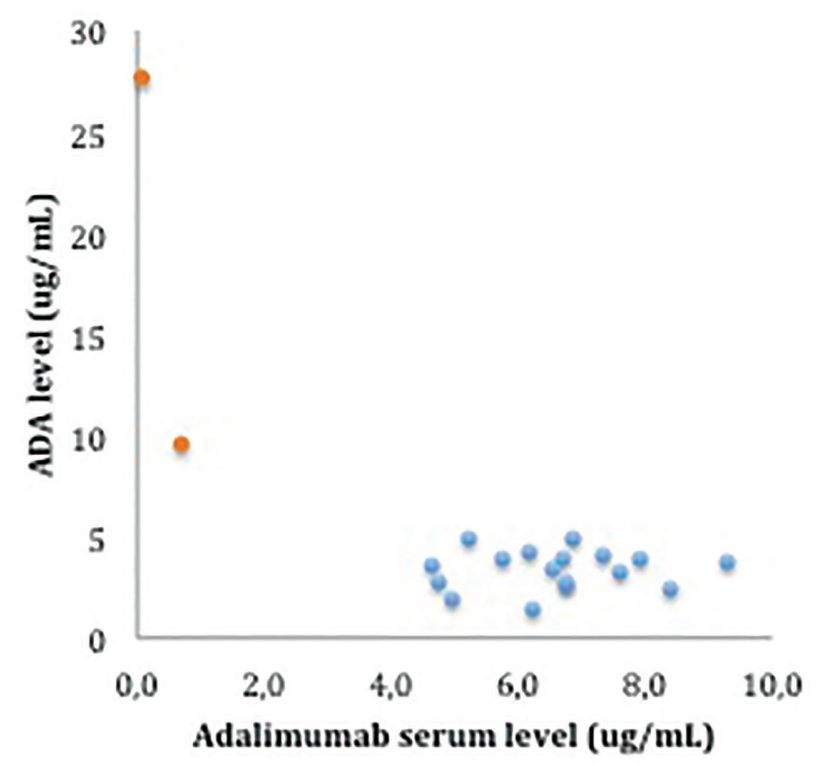

Figure 1 | Distribution of adalimumab and ADA serum levels. Orange dots = partial responders (PASI 50-75), blue dots $=$ responders (PASI > 75), ADAs = anti-drug antibodies.

\section{Discussion}

Immunogenicity is a risk of every treatment based on biological drugs, and may lead not only to loss of response but also to development of adverse events (5). In this work, the detection of low levels of adalimumab and high levels of ADAs using a sandwich ELISA correlated with loss of clinical response. The cost of the treatment for both patients with partial response might have been lower if the measurements had been made earlier. The low number of patients included and consequently the absence of statistical significance represent a limitation of this work.

Although there are data regarding immunogenicity of biological drugs in psoriasis patients, they are mostly from clinical trials, whereas data from real life clinical practice is scant, with reported rates of ADA development in adalimumab-treated psoriasis patients between 6 and 50.9\% (8, 9). In a study by Chiu et al., ADAs were detected in $50.9 \%$ of 53 psoriatic patients treated with adalimumab (8). The differences in PASI75 response rates among patients with and without ADAs were statistically significant (44.4\% vs. $88.5 \%$; $\mathrm{p}=0.001)$. Patients with ADAs had significantly lower trough concentrations of adalimumab than those without ADAs (1.27 vs. $3.55 \mathrm{mg} / \mathrm{l} ; \mathrm{p}=0.02$ ). Another study, by Menting et al., showed a rate of ADA development of $49 \%$ of 80 psoriatic patients treated with adalimumab after a period of 52 weeks (9). The presence of high levels of ADAs correlated with a low adalimumab concentration and lack of clinical response. In our study, two patients $(11 \%)$ developed ADAs. This rate is lower than the studies mentioned; however, the small number of patients and the fact that patients had different treatment durations when the measurements were made might justify this difference. One point that is in agreement with the previous studies is that the presence of high titers of ADAs correlates with low adalimumab concentration and a lack of clinical response.

The main risk factors for ADA development seem to be treatment interruption, absence of concomitant methotrexate use, and biologics switching (6). In addition, low trough adalimumab levels are associated with and likely secondary to the development of ADAs (3).

As for adalimumab serum levels, some studies have aimed to define a therapeutic range that corresponds to an optimal clinical response. Menting et al. established a therapeutic range of adalimumab trough levels of $3.51 \mathrm{mg} / 1$ (or $\mu \mathrm{g} / \mathrm{ml}$ ) to $7.00 \mathrm{mg} / \mathrm{l}$ based on samples from 135 patients (10). In our study, all patients that were responders had serum levels of adalimumab above $3.51 \mu \mathrm{g} /$ $\mathrm{ml}$ (minimum level $4.6 \mu \mathrm{g} / \mathrm{ml}$, Figure 1) and both partial responders had lower levels (Table 2).

These studies are important for establishing protocols for the tailored use of these drugs according to each patient, which might allow considerable cost savings $(10,11)$. This is important not only in patients that do not respond or lose response to biologics, but also in responding patients in which it might be possible to optimize the dose or interval of administration while maintaining efficacy (11). It will be interesting to use data collected from patients with a PASI $>75$ and try to optimize the administration of the drug.

Testing immunogenicity and drug pharmacokinetics of biological drugs in psoriasis patients will probably be part of the daily management of these patients in the near future and should be taken into account alongside other factors such as the clinical efficacy of the drug, the safety profile, and the impact on comorbidities.

\section{References}

1. Vincent FB, Morand EF, Murphy K, Mackay F, Mariette X, Marcelli C. Antidrug antibodies (ADAb) to tumour necrosis factor (TNF)-specific neutralising agents in chronic inflammatory diseases: a real issue, a clinical perspective. Ann Rheum Dis. 2013;72:165-178.
2. Lories RJ, de Vlam K. Tumour necrosis factor inhibitors in the treatment of psoriatic arthritis: a view on effectiveness, clinical practice and toxicity. Expert Opin Biol Ther. 2014;14:1825-36. 
3. Bendtzen K. Immunogenicity of anti-TNF-a biotherapies: I. Individualized medicine based on immunopharmacological evidence. Front Immunol. 2015;6:152.

4. Warren RB, Smith CH, Yiu ZZ, Ashcroft DM, Barker JN, Burden AD, et al. Differential drug survival of biologic therapies for the treatment of psoriasis: a prospective observational cohort study from the British Association of Dermatologists Biologic Interventions Register (BADBIR). J Invest Dermatol. 2015;135:2632-40.

5. Bendtzen K, Geborek P, Svenson M, Larsson L, Kapetanovic MC, Saxne T. Individualized monitoring of drug bioavailability and immunogenicity in rheumatoid arthritis patients treated with the tumor necrosis factor alpha inhibitor infliximab. Arthritis Rheum. 2006;54:3782-9.

6. Gan SD, Patel KR. Enzyme immunoassay and enzyme-linked immunosorbent assay. J Invest Dermatol. 2013;133:e1.

7. Schmidt SD, Mazzella MJ, Nixon RA, Mathews PM. A $\beta$ measurement by enzymelinked immunosorbent assay. Methods Mol Biol. 2012;849:507-27.
8. Chiu HY, Wang TS, Chan CC, Lin SJ, Tsai TF. Risk factor analysis for the immunogenicity of adalimumab associated with decreased clinical response in Chinese patients with psoriasis. Acta Derm Venereol. 2015;95:711-6.

9. Menting SP, van Lumig PP, de Vries AC, van den Reek JM, van der Kleij D, de Jong $E M$, et al. Extent and consequences of antibody formation against adalimumab in patients with psoriasis: one-year follow-up. JAMA Dermatol. 2014;150:130-6.

10. Menting SP, Coussens E, Pouw MF, van den Reek JM, Temmerman L, Boonen H, et al. Developing a therapeutic range of adalimumab serum concentrations in management of psoriasis: a step toward personalized treatment. JAMA Dermatol. 2015;151:616-22.

11. Carrascosa JM. Inmunogenicidad en terapia biológica. Implicaciones en dermatología. Actas Dermosifiliogr. 2013;104:471-9. 